\title{
Research and Design of the Simulation System of ATP System of Quantum satellite
}

\author{
Xin YE1, Qing PAN2\& Zhenghong DONG2 \\ ${ }^{1}$ Department of Graduate Management, Equipment Academy, Beijing 101416, China; \\ ${ }^{2}$ Department of Information Equipment, Equipment Academy, Beijing 101416, China;
}

Keyword: quantum satellite, ATP, Distributed Simulation, IOCP.

\begin{abstract}
Aim to the requirement of the mission of the Tracking and targeting to ground of the quantum communication satellite, this paper investigates an idea of designing a distributed simulation system based on the TCP/IP technology improved by IOCP(I/O Completion Ports). This simulation system is made up of five subsystems and can verify the validity and accuracy of the ATP (Acquisition, Tracking and Pointing) control algorithm of the quantum communications satellite, then show the Real-time operating state of attitude and orbit by ADAMS and STK. This paper analyzes the key technology of IOCP, satellite model building, real-time simulation visualization and simulation synchronization mechanism, and then gives the design scheme of this simulation system, finally, gets the result of simulation.
\end{abstract}

\section{Introduction}

Quantum communication, as a new means of communication, is combined by quantum physics and information theory. It can be divided into fiber channel quantum communication and free space quantum communication. The free space quantum communication has been more researched because of its longer distance and wider range of secure communication. Experiments have verified the feasibility of achieving quantum communication between satellite and ground. So that, it is possible to structure secure and flexible satellite communications system by combining the security of quantum communication and the flexibility of traditional satellite communication together. The ATP technology is the key technology of establishing a communication link between satellite and ground.

Distributed Simulation combined geographically dispersed computers and various simulators and other simulation apparatus organically as a whole via computer network technology, forming a virtual environment of coupling with each other in time and space and interacting with other. [1] This paper is a study of satellite-to-ground quantum key communication ATP system by establishing a distributed simulation software system, while displaying real-time status of satellite attitude and orbit of simulation in two and three dimension.

\section{Components of the Simulation System}

According to the requirement of the satellite-to-ground ATP system of the quantum communication system, the simulation system is divided into five subsystems, namely: satellite platform model subsystem, simulation model of satellite orbit subsystem, the satellite attitude control software subsystem, software interface control subsystem and data analysis subsystem. The system structure is shown below. 


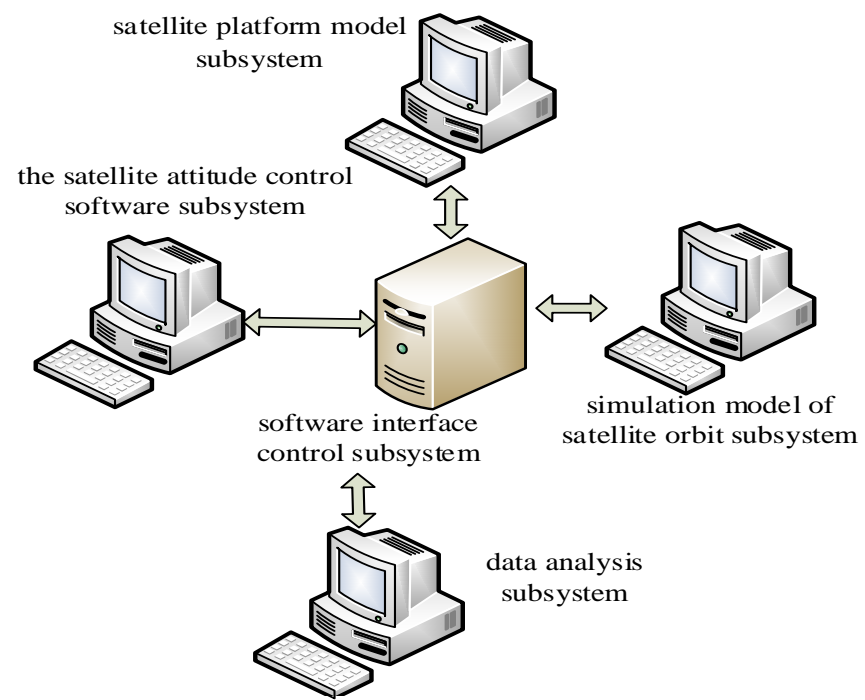

Fig.1: The architecture of the satellite-to-ground ATP simulation system

Among them, the software interface control subsystem is the core hub of the entire simulation system, which connects to the other four subsystems, and plays a role as a server in the simulation system. It completes the interaction of data and instructions between all subsystems, while ensuring the synchronization of simulation time between the subsystems.

The role of satellite platform model subsystem is to establish a satellite entity model and receive attitude control data through interface. The subsystem is also used to do dynamics simulation for the model, and then display the simulation results in real-time three-dimensional, while outputting the satellite platform's and payload's attitude data.

Satellite orbit analysis subsystem is mainly used to build a satellite orbit model and do dynamic simulation, while displaying simulation result in two-dimensional and three-dimensional scene of real-time. At the same time, changing the satellite's attitude in three-dimensional scene by the satellite platform and payload attitude data provided by satellite platform model subsystem to achieve synchronous attitude and orbit simulation.

Satellite attitude control subsystem loads the satellite attitude control algorithms to provide real-time data of dynamic satellite attitude control of model.

Data analysis subsystem provides good interactive features, and is responsible for setting parameters for testing, starting and stopping the system as well as displaying the output data.

\section{Analysis of the Simulation System}

Now, the distributed simulation system are meanly built by using HLA (High Level Architecture) model. HLA has obvious advantages in solving the heterogeneous, distributed, collaborative simulation model and simulation system's interoperability and reusability, which is the preferred construction of large-scale distributed simulation system. But on the other hand, due to the real-time of the current main applications, which named RTI (Run Time Infrastructure) support environment, is not very well, it makes its application being limited in many areas. For the mission requirements and characteristics of the simulation system, it is built based on the TCP / IP protocols with the IOCP model to improve it. Compared with the model simulation system based on HLA, this method spends shorter period of time for a single simulation step of spacecraft orbit.

\section{Design of the Simulation System}

Quantum satellite ATP simulation software system is studied in three levels, namely: the application layer, the system layer and the level of key technology. The research-level framework diagram is below. 


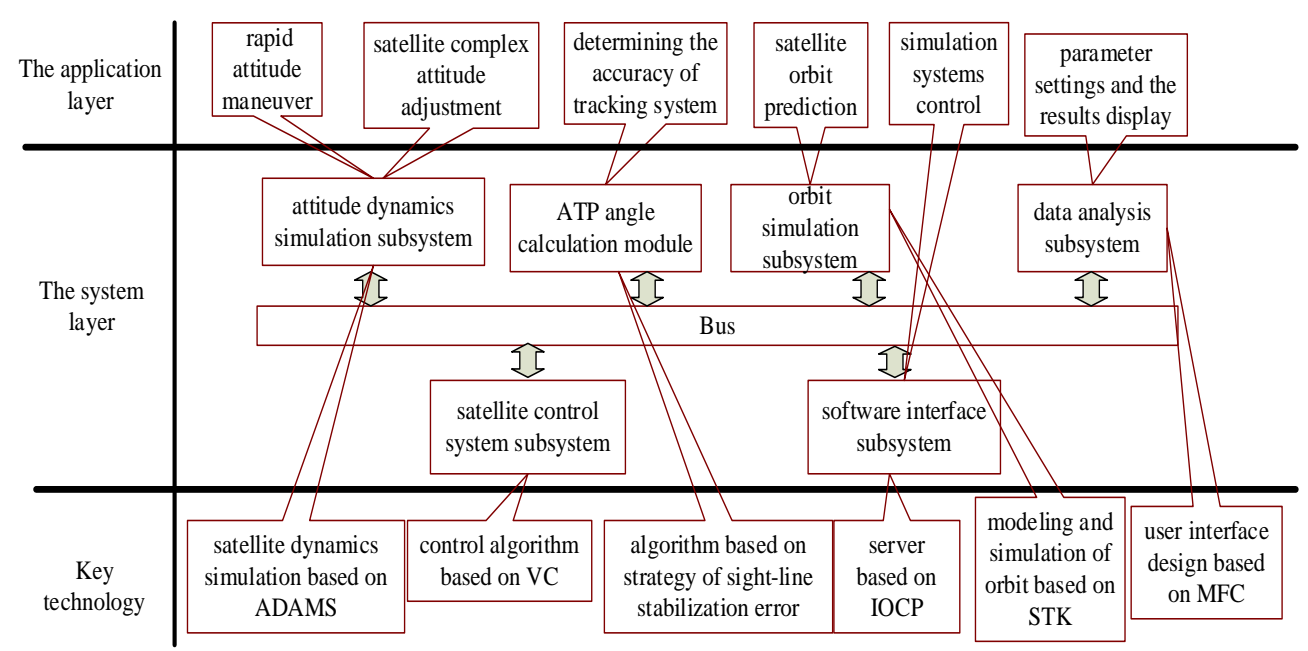

Fig.2: The research-level framework diagram

The application layer: carrying out the whole process of simulation applications of quantum satellites ATP system, including: rapid attitude maneuver, satellite complex attitude adjustment, determining the accuracy of tracking system, satellite orbit prediction, simulation systems control, parameter settings and the results display.

The system layer: In order to support the whole process of simulation of quantum satellite ATP system, the appropriate simulation modules must be established, including: attitude dynamics simulation subsystem, orbit simulation subsystem, satellite control system subsystem, software interface subsystem, data analysis subsystem and ATP angle calculation module.

Key technology: In order to achieve the function of each module, the key technology must be carried out on research, including: satellite modeling based on Pro/E, satellite dynamics simulation based on ADAMS, control algorithms based on VC, algorithm based on strategy of sight-line stabilization error, server based on IOCP, modeling and simulation of orbit based on STK and user interface design based on MFC.

\section{Key technology}

\section{Input / Output Completion Port.}

Input/ Output Completion Port, referred to as IOCP, is a highly efficient I/O model, which use thread pool to handle asynchronous overlapping I/O. It mainly contains an I/O completion event queue and a thread stack. The complete event queue will notify idle threads for data processing. [3] When establishing the complete port model, a unified thread pool should be established at first, and this thread pool is not the same as traditional thread pool which establishes threads when responding to the connection. The number of threads in the thread pool is determined by the number of the CPU core. 


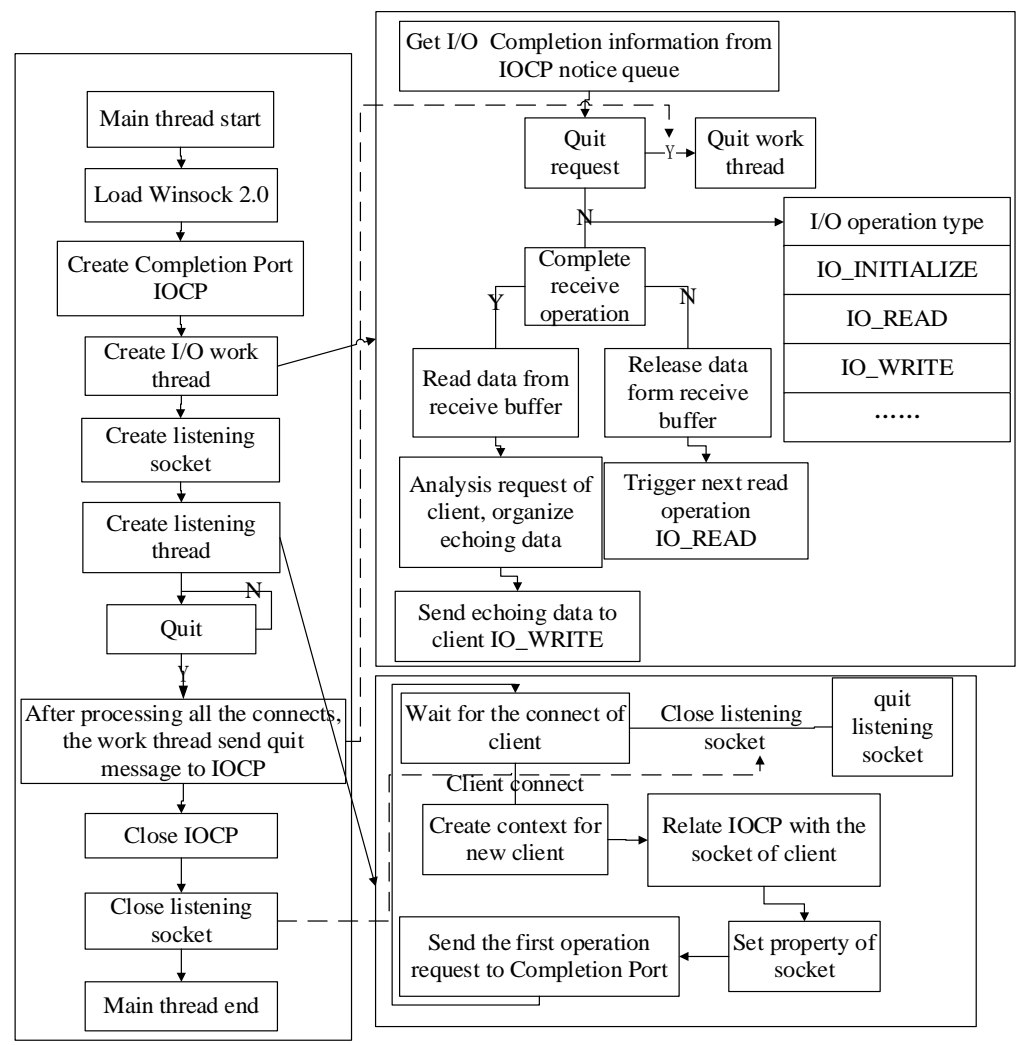

Fig.3: The flowchart of the main thread

IOCP implementation process is as follows: the main thread circularly receives connection requests from clients, while delivering asynchronous I/O requests to clients. After the system completes the I/O operations, the data received will be put into the $\mathrm{I} / \mathrm{O}$ completion event queue, and the queue will call the function remove the I/O operation results from the IOCP, and begin data processing, when the work thread is idle. Then it will delivery I/O requests asynchronously depending on the need of the specific I/O port, and so on until the program exits. [4] The flowchart of the main thread is shown in fig.3.

\section{Simulation Synchronization Mechanism.}

Simulation synchronization mechanism generally is divided into logical synchronization and clock synchronization. Logical synchronization only requires for the order and causality of occurrence of events is strictly aligned, while the local clock of each simulation node is not necessarily exactly the same. Clock synchronization is that each simulation node keep their local clock in the same step.

As a kind of logical synchronization, Satellite orbit analysis subsystem is used to control the simulation synchronous of the whole system. Satellite orbit analysis subsystem gives the satellite propulsion simulation run time, while all subsystems complete their simulation mission synchronously. After reaching the same time, the satellite orbit analysis subsystem continue to give the next time, otherwise the simulation system will remain in the simulation time. Simulation system synchronization control information diagram below.

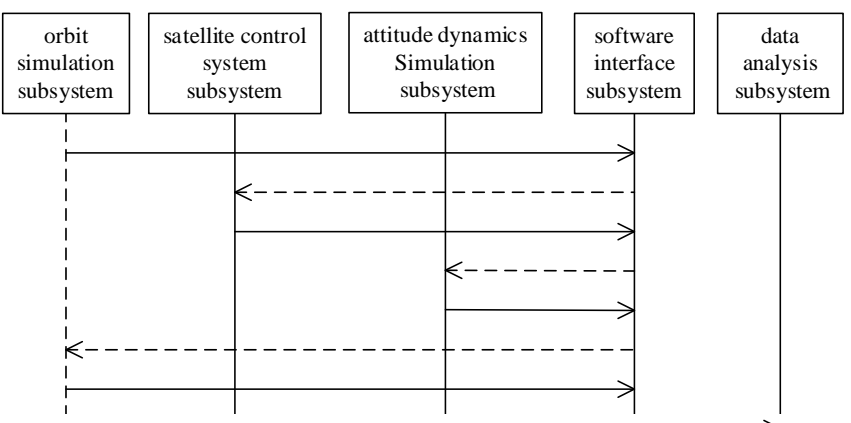

Fig.4: Simulation system synchronization control information diagram 


\section{Satellite Model Building.}

Solid model is the core of the simulation system. By a solid model, real-time operational status of the satellite can be obtained. Solid model of the system is built based on the known parameters of the satellite platform and payload by using Pro/E, and then the model is imported into dynamics simulation software ADAMS, with the appropriate quality, inertia parameters and sports joints, torque and force being set. The interface between ADAMS and matlab/simulink is used to build the external interface of the module, and the satellite model kinematics and dynamics simulation is done by these control commands which are received from satellite control subsystem through this interface to get the real-time satellite attitude data and is displayed through ADAMS/View. The satellite solid model is as follows.

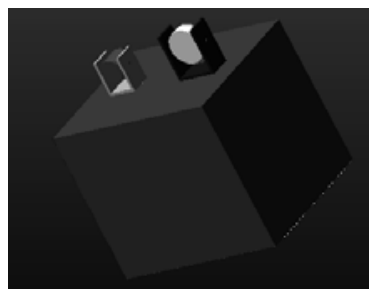

Fig.5: The satellite solid model

\section{Real-time simulation visualization.}

Real-time simulation visualization displays the operating status of the satellite solid model in the form of animation to visually reflect the process of running and can intuitively find problems in the simulation for assisting analysis of simulation results. It is divided into two-dimensional scenes and three- dimensional scene. The former displays orbit satellites running posture in two-dimensional plane, while the latter displays real-time satellite orbit and attitude in three-dimensional scene.

In this paper, the real-time simulation visualization is achieved by the secondary development of the STK/X component. STK/X component is a COM-compliant ActiveX control which is introduced in STK. [5] STK/X component technology allows developers to bring their applications integrating together with STK's two-dimensional display module, three-dimensional display module and data analysis engine, which makes the development of powerful, precise control, well human-computer interaction space simulation applications become possible. Quantum satellite attitude and orbit visualization interface of this paper is as follows.

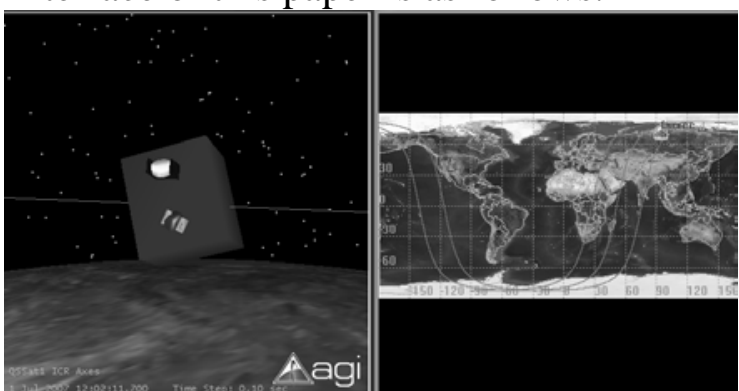

Fig.6: Visualization interface of orbit and attitude of quantum satellite

\section{Simulation Result}

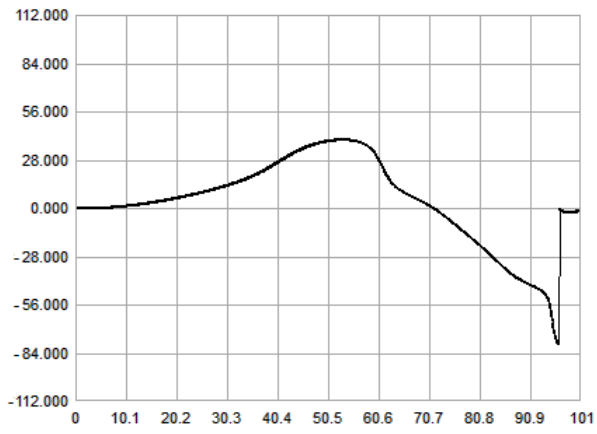

Fig.7: Azimuth of the satellite payload 


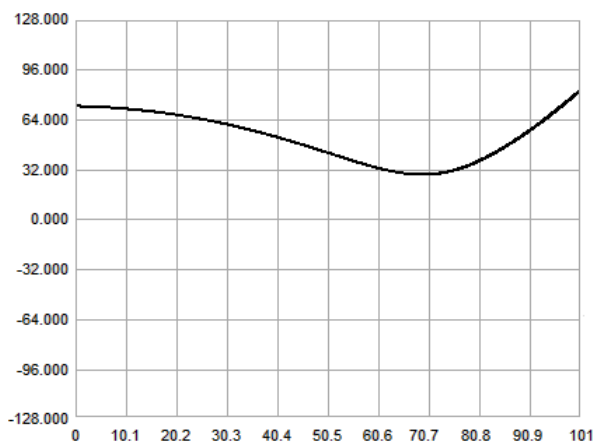

Fig.8: Pitch angle of the satellite payload

Through these Figures, we can see that the range of azimuth angle of the satellite payload changes from -80 to 40 degree, while the range of pitch angle of the satellite payload changes from 30 to 70 degree. The set of data is in line with the quantum satellite-to-ground pointing process.

\section{Conclusion}

According to the ATP mission requirements of the quantum satellite, this paper proposes a design ideas of distributed simulation system based on TCP / IP protocol by using IOCP, and analyzes the system framework and key technology of the simulation system. Finally, this paper gives the simulation result of this distributed simulation system and demonstrates the effectiveness of the simulation system. The design of the distributed simulation system which this paper presents has the following features: real-time; convenient system construction; the simple of technique; no additional environmental support. For building those simulation systems which have fewer nodes or smaller scale have a good reference.

\section{References}

[1] Chen Tao, Gao Wei, Zhang Guo-Yao. Design of Distributed Simulation Based on TCP/IP Protocol. Journal of Projectiles, Rockets, Missiles and Guidance, 28(4), pp.47-52, 2008.

[2] Zhou Jian-Yong, Jiang Zi-Cheng, Wang Yue-Feng, Research on the Aerocraft Maneuver Flighting Distributed Simulation System. Journal of System Simulation, 23(11), pp. 2391-2394, 2011.

[3] Liu Zhen-Yang, Song Yi-Fei, Zhang Rui, Software Design Based on High-powered Server of IOCP. Computer \& Digital Engineering, 41(7), pp.1154-1156, 2013.

[4] Jiang Shu-Xia, Wang Yue, IOCP Application in Server Development. Communications Technologies, 23(3), pp.72-75, 2010

[5] Li Zhi-Qiang, Wu Xi, Rong Ming, Li Xiao-Long, Basics and Applications of STK in System-of-Systems Operations, Beijing: Military Medical Science Press, pp. 302-303, 2014. 